\title{
Spin-orbit-induced gap modification in buckled honeycomb XBi and XBi3 (X = B, Al, Ga, and In) sheets
}

\author{
Rafael R. Q. Freitas, F. de Brito Mota, R. Rivelino, C. M. C. de Castilho, Anelia \\ Kakanakova-Georgieva, Gueorgui Kosto and Gueorguiev
}

\section{Linköping University Post Print}

\section{Tweet}

N.B.: When citing this work, cite the original article.

Original Publication:

Rafael R. Q. Freitas, F. de Brito Mota, R. Rivelino, C. M. C. de Castilho, Anelia KakanakovaGeorgieva, Gueorgui Kosto and Gueorguiev, Spin-orbit-induced gap modification in buckled honeycomb XBi and XBi3 (X = B, Al, Ga, and In) sheets, 2015, Journal of Physics: Condensed Matter, (27), 48, 485306.

http://dx.doi.org/10.1088/0953-8984/27/48/485306

Copyright: IOP Publishing: Hybrid Open Access

http://www.iop.org/

Postprint available at: Linköping University Electronic Press

http://urn.kb.se/resolve?urn=urn:nbn:se:liu:diva-123752 


\title{
Spin-Orbit-Induced Gap Modification in Buckled Honeycomb
}

$$
\mathrm{XBi} \text { and } \mathrm{XBi}_{3}(\mathrm{X}=\mathrm{B}, \mathrm{Al}, \mathrm{Ga} \text {, and } \mathrm{In}) \text { Sheets }
$$

R. R. Q. Freitas ${ }^{1,2}$, F. de Brito Mota ${ }^{1}$, R. Rivelino ${ }^{1}$, C. M. C. de Castilho*,1,3, A. Kakanakova-Georgieva $^{2}$ and G. K. Gueorguiev ${ }^{*}, 2$

${ }^{I}$ Grupo de Física de Superfícies e Materiais, Instituto de Física, Universidade Federal da Bahia, Campus Universitário da Federação, 40170-115 Salvador, Bahia, Brazil;

${ }^{2}$ Department of Physics, Chemistry and Biology (IFM), Linköping University, 58183 Linköping, Sweden;

${ }^{3}$ Instituto Nacional de Ciência e Tecnologia em Energia e Ambiente (CIENAM) INCT-E\&A, Universidade Federal da Bahia, Salvador, Bahia, Brazil

"Corresponding authors: G. K. Gueorguiev (Email: gekos@ifm.liu.se) and C. M. C. de Castilho (Email: caio@ufba.br)

\begin{abstract}
The band structure and stability of $\mathrm{XBi}$ and $\mathrm{XBi}_{3}(\mathrm{X}=\mathrm{B}, \mathrm{Al}, \mathrm{Ga}$, and $\mathrm{In})$ single sheets are predicted using first-principles calculations. It is demonstrated that the band gap values of these new classes of two-dimensional (2D) materials depend on both the spin-orbit coupling (SOC) and type of group-III elements in these hetero-sheets. Thus, topological properties can be achieved, allowing for viable applications based on coherent spin transport at room temperature. The spin-orbit effects are proved to be essential to explain the tunability by group-III atoms. A clear effect of including SOC in the calculations is lifting the spin degeneracy of the bands at the $\Gamma$ of the Brillouin zone. The nature of the band gaps, direct or indirect, is also tuned by SOC, and by the appropriate $\mathrm{X}$ element involved. It is observed that, in the case of XBi single sheets, band inversions naturally occur for $\mathrm{GaBi}$ and $\mathrm{InBi}$, which exhibit band gap values around $172 \mathrm{meV}$. This indicates that these $2 \mathrm{D}$ materials are potential candidates for topological insulators. On the contrary, a similar type of band inversion, as obtained for the $\mathrm{XBi}$, was not observed in the $\mathrm{XBi}_{3}$ band structure. In general, the calculations, taking into account $\mathrm{SOC}$, reveal that some of these buckled sheets exhibit sizable gaps, making them suitable for applications in room-temperature spintronic devices.
\end{abstract}

KEYWORDS: Bismuth, 2D materials, Topological Insulators, DFT, Spin-orbit coupling 


\section{INTRODUCTION}

Intense research in two-dimensional (2D) semiconductor materials has been conducted as prospective graphene alternatives [1-5]. It expands into the group-III nitrides involving the most recognizable example at present, the 2D hexagonal $\mathrm{BN}$ [6-8], but also hexagonal AlN [9-12], and hexagonal GaN [13]. More recently elemental phosphorus has been demonstrated to be stable in different structures [14,15]. It is to be noted that group-III nitrides are valuable semiconductor materials in their wurtzite crystal structure. These materials have been successfully developed by the use of metal organic chemical vapor deposition (MOCVD) for implementation in widely spread energy-saving white lightemitting sources [16].

In the same category of III-V materials, the group-III bismuthides are largely unexplored systems. Indeed, there is a lack of experimental data concerning binary compounds such as $\mathrm{AlBi}, \mathrm{GaBi}$, and $\mathrm{InBi}$. The properties of these materials have been predicted by density functional theory (DFT) calculations of their band and crystalline structure [17]. More recently, the properties of $\mathrm{BBi}, \mathrm{AlBi}, \mathrm{GaBi}, \mathrm{InBi}$, and $\mathrm{TlBi}$, have been also investigated within DFT [18-19]. Only recently thin films of crystalline InBi have been obtained by molecular beam epitaxy [20]. On the other hand, the overall bismuth chemistry (characterized by its low toxicity) and metallurgy is relatively well known, mainly as a result of Bi application as lead replacement in brass alloys [21] and due to the Bi-alloys low-melting points for special solders [22]. Well known in bearings technology is the Al-Bi metallic alloy system [23]. Some Al-Bi-M ( $\mathrm{M}=\mathrm{Cu}, \mathrm{Si}, \mathrm{Sn})$ ternary alloys [24] have also been reported.

An essential factor favoring the research exploration into Bi-containing materials is the availability of Bi-precursors for various deposition techniques, including MOCVD, as employed for depositing bismuth selenides [25], and atomic layer deposition (ALD), as employed for depositing bismuth titanate thin films [26]. While bismuth selenides and tellurides are semiconductors and thermoelectric materials, bismuth titanate, vanadate and other oxides belong to a class of layered perovskite materials, with applications as ferroelectric compounds in electronics and optics [26]. Exfoliated flakes of $\mathrm{Bi}_{2} \mathrm{Se}_{3}, \mathrm{Bi}_{2} \mathrm{Te}_{3}$ and $\mathrm{Bi}_{4} \mathrm{Br}_{4}$ are presently associated with the search of quantum spin Hall insulators [27, 28]. It can be envisaged that, due to its finest control of thickness, attempts for the synthesis of 2D hexagonal equivalents of group-III bismuthides may involve ALD. Accordingly, the selection of proper substrates, those with weak interactions, for example 
$\mathrm{MoS}_{2}$, h-BN, as well as hydrogenated surfaces, and also hydrogenated $\mathrm{SiC}, \mathrm{Si}$ or graphene, are the most promising [29, 30 PRB88 165301].

The 2D hexagonal equivalents of group-III bismuthides are currently an emerging topic [29] in the field of low dimensional materials. First-principles electronic structure calculations predict them to be topological insulators with large enough band gap $(\sim 560$ $\mathrm{meV}$ ) for room temperature applications. In this work, we comparatively investigate two classes of hexagonal group-III bismuthides with stoichiometry of $\mathrm{XBi}$ and $\mathrm{XBi}_{3}(\mathrm{X}=\mathrm{B}$, $\mathrm{Al}, \mathrm{Ga}$ and In). The latter was inspired by recent DFT calculations, emphasizing the interest in the stability and synthesizability of novel 2D hexagonal sheets with a composition $\mathrm{XY}_{3}(\mathrm{X}=\mathrm{B}, \mathrm{C}, \mathrm{N}, \mathrm{Al}, \mathrm{P}$ and $\mathrm{Y}=\mathrm{Si}, \mathrm{C})$ [31-33]. The equilibrium structure of such sheets can be buckled, as in the cases of $\mathrm{NSi}_{3}$ and $\mathrm{PSi}_{3}$, while other $\mathrm{XSi}_{3}(\mathrm{X}=\mathrm{B}$, $\mathrm{C}, \mathrm{Al}$ ) are flat [31]. The versatility of $\mathrm{XY}_{3}$ structures (from buckled at different degrees to completely flat) is complemented by the diversity of their electronic properties. For example, similarly to graphene and silicene, the $\mathrm{CSi}_{3}$ sheet is predicted to be a zero-band gap semi-metal, while other 2D sheets, such as $\mathrm{AlSi}_{3}$ and $\mathrm{PSi}_{3}$, are narrow-band gap semiconductors [31]. These findings make such sheets attractive materials for the emerging 2D materials for nanoelectronics.

By employing DFT calculations, we assess the stoichiometric and geometrical compatibility for the equilibrium structures of hexagonal $\mathrm{XBi}$ and $\mathrm{XBi}_{3}$ sheets. The dynamical stability was evaluated by calculating the phonons spectra as well as the energetic stability for all the studied systems. We also focus on the electronic properties of $\mathrm{XBi}$ and $\mathrm{XBi}_{3}$ exploring the spin-orbit effects on their band structure. This paper is structured as follows: after a description of the calculation methods in Section 2, the results and discussions for $\mathrm{XBi}$ and $\mathrm{XBi}_{3} 2 \mathrm{D}$ sheets are presented in Section 3, while the summary and conclusions represent the content of Section 4.

\section{COMPUTATIONAL DETAILS}

The approaches adopted for all calculations relevant to this work are based on DFT, within its generalized gradient approximation (GGA), as implemented in the VASP code [34]. We employ the Perdew-Burke-Ernzerhof [35] approximation in order to describe the exchange-correlation potentials with the projector augmented wave (PAW) method [36] to describe the electron-ion interaction. This functional has successfully been used to predict the band structures of related Bi-containing layers induced by spin-orbit coupling (SOC) $[29,37]$. As Bi is a heavy atom, the SOC is explicitly taken into account 
in the band structure calculations, by performing fully non-collinear magnetic structure calculations [38] after obtaining the nonmagnetic ground state of the systems.

The hexagonal sheets of $\mathrm{XBi}$ and $\mathrm{XBi}_{3}(\mathrm{X}=\mathrm{B}, \mathrm{Al}, \mathrm{Ga}$ and $\mathrm{In})$ were modelled by employing an hexagonal unit cell containing two and eight atoms, respectively, while the length of the c vector was set to $20 \AA$, so that successive monolayers do not interact along the direction of the c vector. The energy cut-off was set to $700 \mathrm{eV}$ and the adopted kmesh was $25 \times 25 \times 1$, and $16 \times 16 \times 1$ for the $\mathrm{XBi}$ and $\mathrm{XBi}$, respectively. For the structural relaxations of the geometric configurations considered, their constituent atoms were allowed to relax until the forces acting on each atom decrease below the value of 0.01 $\mathrm{eV} / \AA$. The total energy convergence criterion, in both cases, was set to be $10^{-5} \mathrm{eV}$. As a complement necessary for assessing the dynamic stability of relaxed $\mathrm{XBi}$ and $\mathrm{XBi}_{3}$ sheets, their phonon spectra were obtained by employing the Density Functional Perturbation Theory (DFPT) method as implemented in the VASP combined with the Phonopy code [39].

Within the adopted scheme, the cohesive energies associated to these $2 \mathrm{D}$ systems were also calculated. Cohesive energy per atom $\left(E_{c o h / a t}\right)$ is defined as follows:

$$
E_{\text {coh } / a t}=-\frac{1}{N}\left(E_{A B}-n_{A} E_{A}-n_{B} E_{B}\right)(1)
$$

In eq. $1, E_{A}$ and $E_{B}$ are the total energies of the free atoms, $E_{A B}$ is the total energy per unit cell of the compound $\mathrm{AB}$, with $n_{A}$ and $n_{B}$ being the corresponding numbers of atoms of each element $\mathrm{A}$ and $\mathrm{B}$ per unit cell and $N$ is the total number of atoms. In the case of hexagonal sheets of $\mathrm{XBi}, n_{A}=n_{B}=1$, while in the case of hexagonal sheets of $\mathrm{XBi}_{3}$, $n_{B}=3 n_{A}$.

\section{RESULTS AND DISCUSSION}

\subsection{Structure and dynamic stability of the XBi and XBiz sheets}

We have obtained, after full relaxation, two equilibrium geometries with a distinct buckling parameter, $\Delta$, graphically defined in figure 1(a), as and displayed in figure 1(b), in accordance with ref. [29]. First, we discuss the structural properties of the hexagonal sheets of $\mathrm{XBi}(\mathrm{X}=\mathrm{B}, \mathrm{Al}, \mathrm{Ga}$, and $\mathrm{In})$, as shown in figure 1 (a). We have obtained, after full relaxation, two equilibrium geometries with a distinct $\Delta$, in accordance with ref. [29]. With the exception of $\mathrm{BBi}$, all the other structures can be classified as low buckled (LB) or high buckled (HB) [40], with, in our systems, the HBs being slightly more favorable than the LB ones. Additionally, we have calculated the phonon spectra of all the XBi 
sheets. Only the LB systems are dynamically stable as shown in figure 1(c) for $\mathrm{GaBi}$, which is, in some extent, rather similar to the phonon spectra of buckled blue phosphorus [15]. On the contrary, the HB structures present imaginary frequencies, as shown in figure 1(d) also for the GaBi. In this context, our results indicate that all four hexagonal sheets of $\mathrm{BBi}, \mathrm{AlBi}, \mathrm{GaBi}$, and InBi represent $\mathrm{LB}$ systems.

The structural parameters of the LB equilibrium structures of all XBi sheets are listed in Table 1 with their lattice parameters varying in the range of $3.89-4.80 \AA$. In terms of both lattice constants and buckling parameters, our results are in excellent agreement with those obtained for the same systems, at a similar level of theory [29]. Also in Table 1, for comparative purposes and to put the $\mathrm{XBi}$ sheets in an appropriate context, the structural parameters of graphene, silicene and h-BN, calculated at the same level of theory, are also listed. At equilibrium, the buckling parameter $\Delta$ varies in the range $0.51-0.85 \AA$. These values of the buckling parameter fall in the range of values obtained for other 2D sheets [41].

As expected, the lattice constant (a) and the buckling parameter $(\Delta)$, gradually increase with the atomic number/atomic size of the group-III element involved. The values vary from $\mathrm{a}=3.89 \AA(\mathrm{BBi})$ to $\mathrm{a}=4.80 \AA$ ( $\mathrm{InBi})$, and $\Delta=0.51 \AA$ (BBi) to $\Delta=0.85$ $\AA$ (InBi). Our results demonstrate that, in terms of structure, the LB sheets of BBi, AlBi, $\mathrm{GaBi}$, and InBi are quite similar among them. This makes them compatible systems, from a point of view of hetero-structures, as well as possible layered materials and assemblies built upon different 2D templates [42]. For example, substrate such as $\mathrm{MoS}_{2}, \mathrm{~h}-\mathrm{BN}$ are the most promising template for doing this [29, 30]

Table 1 also lists the cohesive energies per atom $\left(E_{\text {coh/atom }}\right)$ of all hexagonal sheets of XBi. As expected, cohesive energies of the LB XBi sheets $(3.25-2.15 \mathrm{eV})$ are lower than the ones calculated at the same level of theory for graphene $(7.83 \mathrm{eV})$, and h-BN $(7.12 \mathrm{eV})$. However, they are comparable to the cohesive energy of silicene $(3.98 \mathrm{eV})$. As in the case of silicene, a possible way of assisting the synthesis of stable XBi sheets would be its growth upon an appropriate substrate [43-44].

In the following, we discuss the structural properties of the hexagonal sheets of $\mathrm{XBi}_{3}(\mathrm{X}=\mathrm{B}, \mathrm{Al}, \mathrm{Ga}$, and $\mathrm{In})$. Due to their more complex eight-atom unit cells, the $\mathrm{XBi}_{3}$ sheets are characterized by lattice parameters in the range of $8.17-9.12 \AA$ (Table 1). The equilibrium structure for the case of $\mathrm{GaBi}_{3}$ is displayed in figure 2(a). At equilibrium, the $\mathrm{XBi}_{3}$ sheets can be structurally represented as two inter-penetrating sublattices, consisting of atoms $\mathrm{X}$ and $\mathrm{Bi}$, respectively, and described by the respective buckling parameters $\Delta_{1}$ 
and $\Delta_{2}$, each one associated with the sublattices as shown in the bottom of figure 2(a). For these two buckling parameters, $\Delta_{1}$ and $\Delta_{2}$, a trend similar to the one discussed above for the sheets of $\mathrm{XBi}$ is also observed. However, the increase in their value with the atomic number/atomic size of the group-III element involved is less pronounced. Another finding in this computational study is that the sublattice corresponding to the group-III elements is significantly less buckled $\left(\Delta_{1}=0.48-0.69 \AA\right)$ than the sublattice consisting of Bi atoms $\left(\Delta_{2}=1.84-1.88 \AA\right)$, which apparently plays a role of a "framing" sublattice of the $\mathrm{XBi}_{3}$ sheets. This behavior is similar to the one observed for the cases $\mathrm{NSi}_{3}$ and $\mathrm{PSi}_{3}$ [31-33].

The phonon spectra of all equilibrium structures of the $\mathrm{XBi}_{3}$ systems were also calculated. Figure 2(b) displays the phonon spectrum of $\mathrm{GaBi}_{3}$, as an example illustrating the absence of any negative frequencies of significant intensity. This confirms that all the $\mathrm{XBi}_{3}$ sheets are dynamically stable. We emphasize that these findings are similar to those previously obtained for HB lead and tin layers [45].

Table 1 also lists the structural parameters, as well as the $\mathrm{X}$-Bi and the $\mathrm{Bi}-\mathrm{Bi}$ bond lengths, corresponding to the equilibrium structures of all $\mathrm{XBi}_{3}$ sheets. In terms of buckling, the $\mathrm{XBi}_{3}$ sheets exhibit a similar behavior (albeit more buckled) when compared to the buckling of the $\mathrm{XBi}$ sheets. Still, the fully relaxed structures of the $\mathrm{BBi}_{3}, \mathrm{AlBi}_{3}$, $\mathrm{GaBi}_{3}$ and $\mathrm{InBi}_{3}$ sheets remain of the LB type.

The cohesive energy calculated for the $\mathrm{XBi}_{3}$ (Table 1 ) indicates that the sheets of $\mathrm{BBi}_{3}, \mathrm{AlBi}_{3}$ and $\mathrm{InBi}_{3}$ exhibit similar energetic stability, albeit with a slightly higher $\mathrm{E}_{\text {coh/at }}$, in comparison to the corresponding sheets of $\mathrm{BBi}, \mathrm{AlBi}$, and $\mathrm{InBi}$. This is especially pronounced in the case of $\mathrm{B}$ and $\mathrm{In} . \mathrm{BBi}$ and $\mathrm{BBi}_{3}$ exhibit cohesive energies of 3.25 and $2.80 \mathrm{eV}$, respectively, whereas $\mathrm{InBi}$ and $\mathrm{InBi}_{3}$ exhibit cohesive energies of 2.15 and $1.89 \mathrm{eV}$, respectively. On the other hand, when comparing the $\mathrm{XBi}_{3}$ with $\mathrm{XBi}$ sheets containing the III-elements with intermediate atomic numbers, i. e., $\mathrm{X}=\mathrm{Al}$ and $\mathrm{Ga}$, we found that they exhibit much closer cohesive energies (see Table 1). The slightly less favorable energetic behavior of $\mathrm{XBi}_{3}$, as compared to $\mathrm{XBi}$ (with exception of the $\mathrm{GaBi}$ ), may be attributable to different bonding arrangements, due to the different composition of $\mathrm{XBi}_{3}$ and $\mathrm{XBi}$, as well as to the presence of homo-nuclear $\mathrm{Bi}-\mathrm{Bi}$ bonds in $\mathrm{XBi}_{3}$.

\subsection{Electronic structure and topological properties of the $\mathrm{XBi}$ and $\mathrm{XBi}$ sheets}

The partial densities of states (PDOS) and corresponding band structures, calculated without spin-orbit coupling (SOC) and with SOC, for the equilibrium structures of all considered sheets of $\mathrm{XBi}$ and $\mathrm{XBi}_{3}(\mathrm{X}=\mathrm{B}, \mathrm{Al}, \mathrm{Ga}$, and $\mathrm{In})$, are displayed 
in figures 3 and 4, respectively. Quantitative differences among the systems arise from the contributions of the $p$ states of $\mathrm{Bi}$ and $\mathrm{X}$ atoms to the PDOS near the Fermi level. Although these systems are structurally similar to silicene and germanene [40-46], the electronic structures are significantly different, leading to a tunable band gap opening, instead of forming gapless systems.

The corresponding band gaps, obtained without and with SOC, are listed in Table 1. As can be seen from figures 3(a)-(d), within the XBi class before including SOC in the calculations, the band structures of the distinct compounds appear qualitatively similar, exhibiting parabolic bands at the $\Gamma$ point, although the band gap varies from 100 up to $750 \mathrm{meV}$. In fact, it is known that, when one employs the Heyd-Scuseria-Ernzerhof hybrid functional, these band gaps may significantly be increased but resulting in similar band structures $[37,48]$. Additionally, these XBi systems exhibit direct band gaps, at the $\Gamma$ point in the cases for $\mathrm{Al}$ to $\mathrm{In}$ and at the $\mathrm{K}$ point for $\mathrm{B}$. Similarly, the Bi-containing topological insulators $\mathrm{Bi}_{2} \mathrm{Te}_{3}$ and $\mathrm{Bi}_{2} \mathrm{Te}_{2} \mathrm{Se}$ have recently been obtained as direct-gap semiconductors [48], when SOC is not taken into account. However, by including SOC, these materials exhibit dramatic changes in the band structures, indicating possible applicability connecting thermoelectric performance and topological insulating properties.

In the sense of investigating and classifying possible topological insulating characteristics of the $\mathrm{XBi}$ and $\mathrm{XBi}_{3}$ sheets, we have also considered the inclusion of $\mathrm{SOC}$ into the electronic structure calculations. Chuang and collaborators [29] have previously observed topological properties only for the XBi family. We have analyzed the s-orbitals projection in the wave function in the band structures for all these systems. In the case of XBi sheets, the observed band inversion could be qualitatively employed to classify their topological-insulating nature. As a typical example of 2D topological insulator, we display in figure 5(a), we display the band structure of $\mathrm{GaBi}$, without and with SOC, where it is demonstrated that the contribution of the s-orbitals moves from the bottom of the conduction band to higher energies. For the buckled equilibrium XBi structures, the band inversion was observed only in the $\mathrm{GaBi}$ and $\mathrm{InBi}$ hetero-sheets. On the other hand, a similar band inversion was not observed in the $\mathrm{XBi}_{3}$ compounds, since there is no contribution of s-orbitals at the bottom of their respective conduction bands. In figure 5 (b), we illustrate the case of $\mathrm{GaBi}_{3}$, although this behavior arises for all $\mathrm{XBi}_{3}$ sheets. Further insight can be obtained from the band inversion strength, which is defined as the 
direct band gap at the $\Gamma$ point, being positive when the band is inverted and negative otherwise [29]. As displayed in figure 6, only $\mathrm{GaBi}$ and InBi present negative values for this quantity. We noticed that the same analysis can not be applied to the $\mathrm{XBi}_{3}$ sheets since they do not present band inversion at the $\Gamma$ point.

We have also noticed first that the band structures of XBi appear modified by spinorbit effects and, especially, the band degeneracy is broken at the $\Gamma$ point. Nonetheless, the calculated band structures with SOC continue to exhibit comparatively parabolic band edges. Now, we find the band gaps to be indirect and varying from 170 up to $480 \mathrm{meV}$. More interestingly, we have observed a small reduction $(\sim 6 \%)$ in the band gap of BBi and a strong reduction $(\sim 55 \%)$ in the band gap of AlBi. Conversely, we have observed a large increase $(\sim 37 \%)$ in the band gap of $\mathrm{GaBi}$ and a small increase $(3 \%)$ in the band gap of InBi. We also emphasize that one of the effects of including SOC in the calculations is also lifting the spin degeneracy [49] of the calculated bands for the XBi systems, as shown in figure (3). Based on the electronic structure, the changes produced by SOC effects make the XBi sheets potential candidates to be exploited as topologically insulating 2D materials.

In turn, when the electronic properties of the $\mathrm{XBi}_{3}$ sheets are calculated without considering SOC, most of them appear as narrow-band-gap semiconductors. For example, $\mathrm{BBi}_{3}, \mathrm{AlBi}_{3}$, and $\mathrm{GaBi}_{3}$ exhibit very small band gaps of less than $80 \mathrm{meV}$, while the $\mathrm{InBi}_{3}$ sheet opens a small band gap of $169 \mathrm{meV}$ (see Table I and figure (4)). With the exception of $\mathrm{BBi}_{3}$, the $\mathrm{XBi}_{3}$ sheets exhibit band degeneracies at the $\Gamma$ point with comparatively parabolic bands, as in the case of $\mathrm{XBi}$. We also noticed that the $\mathrm{XBi}_{3}$ sheets only exhibit an indirect band gaps in the cases of $\mathrm{X}=\mathrm{B}, \mathrm{Al}$ and $\mathrm{Ga}$. By including SOC into the calculations, in general larger band gaps of the $\mathrm{XBi}_{3}$ sheets are obtained; i.e., 526, 263, 286, and $244 \mathrm{meV}$ for the $\mathrm{BBi}_{3}, \mathrm{AlBi}_{3}, \mathrm{GaBi}_{3}$, and $\mathrm{InBi}_{3}$, respectively. In addition, the inclusion of SOC removes the band degeneracies at the $\Gamma$ point, with the exception of $\mathrm{BBi}$, which as already pointed out, does not exhibit this degeneracy. This may be the reason for the enormous increase on the band gap in the case of BBi upon SOC inclusion. Another peculiarity of $\mathrm{BBi}$ is that it presents the higher value for the $\Delta_{1}$ buckling parameter, breaking the planarity of the $\mathrm{B}-\mathrm{Bi}$ bonds. The nature of the band gap, direct or indirect, is not affected by inclusion of SOC, with the exception of $\mathrm{AlBi}_{3}$, which becomes now an indirect-gap semiconductor. 


\section{CONCLUSIONS}

From first-principles calculations, the sheets of $\mathrm{XBi}$ and $\mathrm{XBi}_{3}$ emerge as two classes of energetically and dynamically stable 2D materials, consisting of group-III elements and bismuth. Similarly to the case of other 2D sheets, the considered $\mathrm{XBi}(\mathrm{X}=$ $\mathrm{B}, \mathrm{Al}, \mathrm{Ga}, \mathrm{In}$ ) sheets exhibit low-buckled equilibrium geometries, while the $\mathrm{XBi}_{3}$ can be structurally represented as two interpenetrating sublattices, consisting of alternating $\mathrm{X}$ and $\mathrm{Bi}$ atoms. The dynamical stability, combined with the tunable electronic properties (by changing the $\mathrm{X}$ element involved) of the $\mathrm{XBi}$ and $\mathrm{XBi}_{3}$ sheets, renders them as being prospective candidates for applications in room-temperature spintronic devices.

With respect to the electronic properties of these materials, the band structures are strongly affected by including SOC into the calculations. Without SOC, the band structures of the $\mathrm{XBi}$ and $\mathrm{XBi}_{3}$ hetero-sheets are qualitatively similar, exhibiting bulk gaps in the 100-750 meV range for $\mathrm{XBi}$ and 5-170 meV range for $\mathrm{XBi}_{3}$. The inclusion of SOC into the calculations keeps a similarity among the band structures by changing the $\mathrm{X}$ element, although it leads to a band degeneracy breaking at the $\Gamma$ point for most of the considered cases, and a large band gap opening for the $\mathrm{XBi}_{3}$ sheets.

In all the cases of $\mathrm{XBi}, \mathrm{SOC}$ changes the band gaps from direct to indirect, varying from 170 up to $480 \mathrm{meV}$, with a small reduction in the case of $\mathrm{BBi}$ and a strong one in the case of AlBi. Nonetheless, there is a large increase in the band gaps of GaBi and a small one in the case of InBi. Additionally, SOC causes the lifting in the spin degeneracy of the calculated bands for the XBi sheets. The band inversion calculated for the $\mathrm{GaBi}$ and $\mathrm{InBi}$ sheets indicates that they naturally represent 2D topological insulating phases. Since DFT is known to underestimate calculated band gaps, we stress that $\mathrm{GaBi}$ and $\mathrm{InBi}$ exhibit sizable gaps, in a range of $170-175 \mathrm{meV}$; i. e., sufficiently large for practical applications at room temperature.

For $\mathrm{XBi}_{3}$, our calculations without SOC show that most of them are small gap semiconductors. Inclusion of SOC into the calculations causes significant band gap openings to a range of 240 to $530 \mathrm{meV}$ in these systems. In this case there is no band inversion as observed in the $\mathrm{XBi}$ compounds, since there is no contribution of s-orbitals at the bottom of their conduction bands. However, it is hoped that unusual properties can emerge from these structures, e.g., in electron transport applications in low dimensions, mainly due to the increase of electronic states near the conduction band. 


\section{ACKNOWLEDGMENTS}

The authors gratefully acknowledge partial financial support for this work by the Swedish Research Council (VR) through Swedish Research links project 348-2014-4249. G.K.G. and A. K. G. gratefully acknowledge support by the Linköping Linnaeus Initiative for Novel Functionalized Materials (LiLi-NFM, VR). G. K. G. acknowledges support by the Swedish Foundation for Strategic Research (SSF) Synergy Grant \#RMA11-0029 on Functional Carbides and Advanced Surface Engineering (FUNCASE). R.R., C.M.C.deC., and F.deB.M. acknowledge ConselhoNacional de DesenvolvimentoCientífico e Tecnológico (CNPq) and Fundação de Amparo à Pesquisa do Estado da Bahia (FAPESB) for partial support. R.R.Q.F. acknowledges the support by Coordenação de Aperfeiçoamento de Pessoal de Nível Superior (CAPES). We acknowledge the fruitful discussions with Dr. S. Cunha during the early stages of this work.

\section{References}

[1] Zhang S et al 2015 Angew. Chem. Int. 541

[2] Rasche B et al 2013 Nature Mater. 12422

[3] Li Zet al 2015 Nature Comm. 66321

[4] Zhang S et al 2015 Proc. Natl. Acad. Sci. USA 1122372

[5] Balendhran S et al 2015 Small 11640

[6] Azevedo S et al 2009 Eur. Phys. J. B 67507

[7] Pakdel A, Bando Y and Golberg D 2014 Chem. Soc. Rev. 43934

[8] Fazzio A et al 2012 J. Phys. Condens. Matter. 24075301

[9] de Almeida Junior E F et al 2012 Eur. Phys. J.B 85, 48

[10] Tsipas P et al 2013Appl. Phys. Lett.103 251605

[11] S. Valedbagi S, Fathalian A and Elahi S M 2013 Opt. Comm. 309153

[12] Le M-Q 2014 J. Comput. Theor. Nanoscience 111458

[13] Li H et al 2010 J. Phys. Chem. C 11411390

[14] Liu H et al 2014 ACS Nano 84033

[15] Zhen Z and Tománek D 2014 Phys. Rev. Lett. 112176802

[16] "The Nobel Prize in Physics 2014 - Advanced Information" 2015 Nobelprize.org.

Nobel Media AB

<http://www.nobelprize.org/nobel_prizes/physics/laureates/2014/advanced.html>

[17] Janotti A, Wei S-H and Zhang S B 2002 Phys. Rev. B 65115203 
[18] Ferhat M and Zaoui A 2006 Appl. Phys. Letters 88161902

[19] Ferhat M and A. Zaoui 2006 Phys. Rev. B 73, 115107

[20] Keen B et al 2014 Journal of Electronic Materials 43914

[21] La Fontaine A and Keast V J 2006 Mater. Charact. 57424

[22] Witkin D 2012 J. Electron. Mater. 41190

[23] Uenishi K, Hayong K and Kobayashi K F1996 J. Mater. Sci. 313605

[24] Kaban I G and Hoyer W 2008 Phys. Rev. B 77125426

[25] Waters J et al 2003 J. Mater. Sci.: Mater. Electro. 14599

[26] Vehkamäki M T et al 2006 Chem. Mater. 183883

[27] Zhou J-J et al 2014 Nano Lett.14 4767

[28] Zhu Z-H et al 2014 Phys. Rev. Lett. 112076802

[29] Chuang F-C et al 2014 Nano Lett.14 2505

[30] Huang Z-Q et al 2013 Phys. Rev. B 88165301

[31] Ding Y and Wang Y 2013 J. Phys. Chem. C 117, 18266

[32] Luo X et al 2011 J. Am. Chem. Soc. 13316285

[33] Ding Y and Wang Y 2014 J. Phys. Chem. C 1184509

[34] Kresse G and J. Hafner 1993 Phys. Rev. B 47 558; Kresse G and Furthmüller 1996 Phys. Rev. B1996 5411169

[35] Perdew J P. K. Burke and Ernzerhof M 1996 Phys. Rev. Lett. 773865

[36] Kresse G and Joubert D 1999 Phys. Rev. B 591758

[37] Ma Y et al 2015 Phys. Rev. B 91235306

[38] Hobbs D et al 2000 Phys. Rev. B 621156

[39] Togo A, Oba F and Tanaka I 2008 Phys. Rev. B 78134106

[40] Cahangirov S et al 2009 Phys. Rev. Lett.102, 236804

[41] Sahin H et al 2009 Phys. Rev. B 80155453

[42] Gao G et al 2012Nano Lett.12 3518

[43] Lalmi B et al 2010 Appl. Phys. Lett. 97223109 and 2010 Phys. Lett. 97223109

[44] Fleurence A et al 2012 Phys. Rev. Lett. 108245501

[45] Rivero P et al 2014 Phys. Rev. B 90 241408(R)

[46] Pan Y et al 2014 Small 102215

[47] Peralta J E et al 2006 Phys. Rev. B 74073101

[48] Shi H et al 2015 Phys. Rev. Appl. 3014004

[49] Ma D, Li Z and Yang Z 2012 Carbon 50297 


\section{Tables}

TABLE I. Calculated structural parameters (in $\AA$ ), cohesive energies per atom (in eV), and energy gaps (in meV) for the $\mathrm{XBi}$ and $\mathrm{XBi}_{3}(\mathrm{X}=\mathrm{B}, \mathrm{Al}, \mathrm{Ga}, \mathrm{In})$ relaxed sheets. Results for graphene, silicene and h-BN are provided at the same level of calculations for comparison.

\begin{tabular}{|c|c|c|c|c|c|c|c|c|}
\hline $\begin{array}{c}\text { 2D } \\
\text { system }\end{array}$ & $\begin{array}{c}\text { Buckling } \\
\text { Type }\end{array}$ & $\mathbf{a}$ & $\begin{array}{c}\Delta \text { or } \\
\Delta_{1} ; \Delta_{2}\end{array}$ & $d_{B i-X}$ & $\mathbf{d}_{\mathbf{B i}-B \mathbf{i}}$ & $\mathbf{E}_{\text {coh/at }}$ & $\mathbf{E}_{\mathbf{g}}$ & $\mathbf{E}_{\mathrm{g}-\mathrm{SOC}}$ \\
\hline graphene & planar & 2.46 & - & - & - & 7.83 & - & - \\
\hline silicene & LB & 3.87 & 0.45 & - & - & 3.98 & - & - \\
\hline h-BN & planar & 2.51 & - & - & - & 7.12 & $4680^{\mathrm{b}}$ & - \\
\hline \multirow[t]{2}{*}{$\mathrm{BBi}$} & LB & 3.89 & 0.51 & 2.30 & - & 3.25 & $511^{\mathrm{b}}$ & $479^{c}$ \\
\hline & & $3.891^{\mathrm{a}}$ & $0.51^{\mathrm{a}}$ & & & & & \\
\hline \multirow[t]{2}{*}{$\mathrm{AlBi}$} & LB & 4.53 & 0.77 & 2.73 & - & 2.56 & $751^{\mathrm{b}}$ & $335^{\mathrm{c}}$ \\
\hline & & $4.523^{\mathrm{a}}$ & & & & & & \\
\hline \multirow[t]{2}{*}{$\mathrm{GaBi}$} & LB & 4.52 & 0.79 & 2.73 & - & 2.31 & $107^{b}$ & $169^{c}$ \\
\hline & & $4.521^{\mathrm{a}}$ & & & & & & \\
\hline \multirow[t]{2}{*}{$\mathrm{InBi}$} & LB & 4.80 & 0.85 & 2.90 & - & 2.15 & $169^{b}$ & $175^{\mathrm{c}}$ \\
\hline & & $4.805^{\mathrm{a}}$ & $0.85^{\mathrm{a}}$ & & & & & \\
\hline $\mathrm{BBi}_{3}$ & buckled & 8.17 & $0.69 ; 1.84$ & 2.33 & 3.06 & 2.80 & $5^{c}$ & $526^{c}$ \\
\hline $\mathrm{AlBi}_{3}$ & buckled & 8.83 & $0.48 ; 1.85$ & 2.77 & 3.04 & 2.45 & $80^{c}$ & $263^{b}$ \\
\hline $\mathrm{GaBi}_{3}$ & buckled & 8.82 & $0.56 ; 1.86$ & 2.77 & 3.03 & 2.33 & $81^{c}$ & $286^{c}$ \\
\hline $\mathrm{InBi}_{3}$ & buckled & 9.12 & $0.58 ; 1.88$ & 2.96 & 3.03 & 1.89 & $169^{\mathrm{b}}$ & $244^{\mathrm{b}}$ \\
\hline
\end{tabular}

${ }^{\text {a) }}$ Results as obtained in Ref. [26]

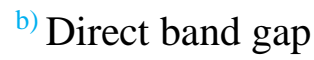

${ }^{c)}$ Indirect band gap 


\section{Figures}

Figure 1. (a)Top and side views of the LB equilibrium structure of the $\mathrm{GaBi}$ sheet $(\Delta=0.79$ $\AA$ );(b) Variation of the total energy with respect to the lattice constant for the $\mathrm{XBi}(\mathrm{X}=\mathrm{B}$, $\mathrm{Al}, \mathrm{Ga}, \mathrm{In}$ ) sheets; (c) Phonon spectra corresponding to the LB GaBi sheet; and(d) Phonon spectra for the HB GaBi sheet.
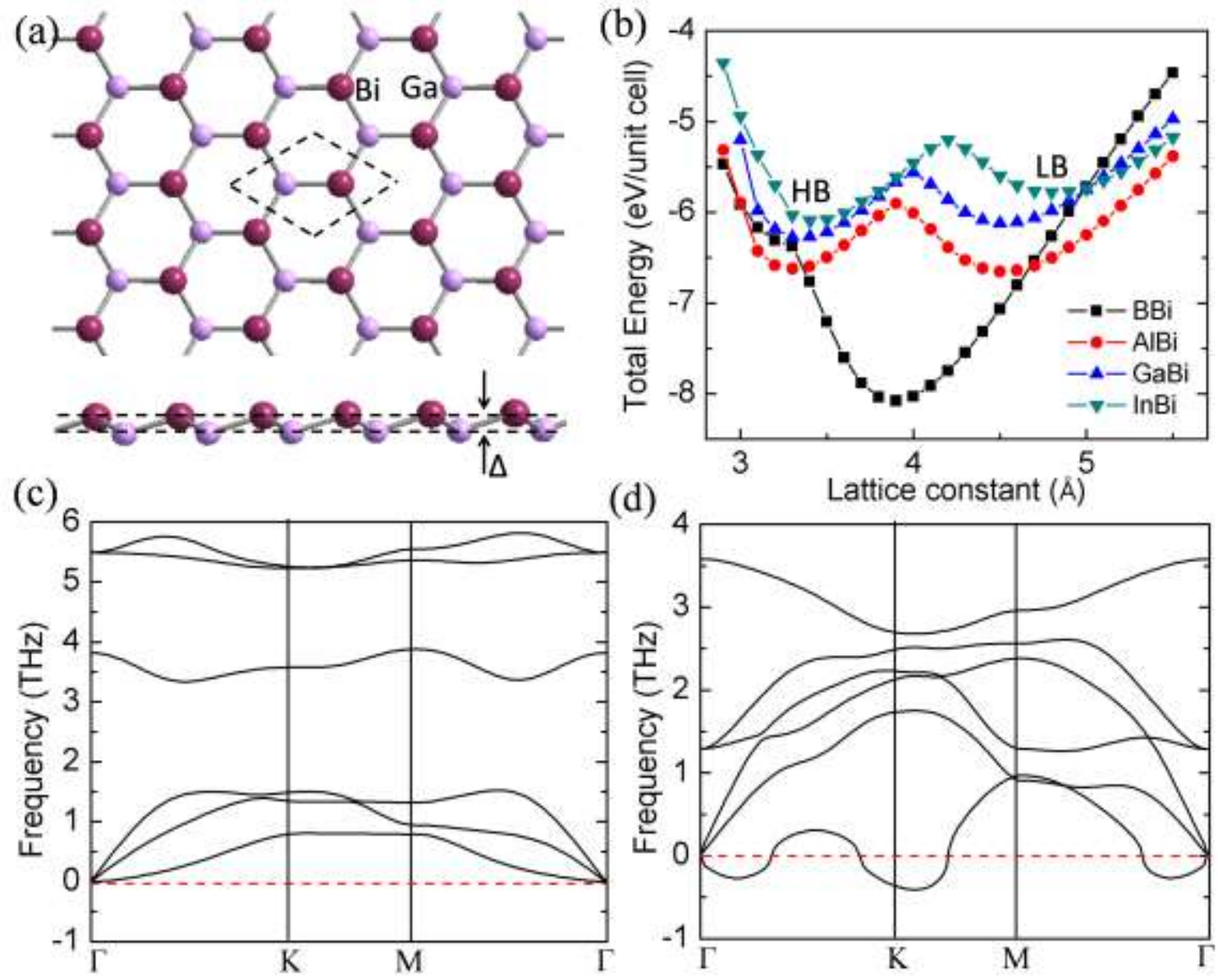
Figure 2. (a) Top and side views of the equilibrium structure of the $\mathrm{GaBi}_{3}$ sheet $\left(\Delta_{1}=\right.$ $0.56 \AA, \Delta_{2}=1.86 \AA$ ); (b) Phonon spectra corresponding to theGaBi 3 sheet. Fermi level was set to zero energy.
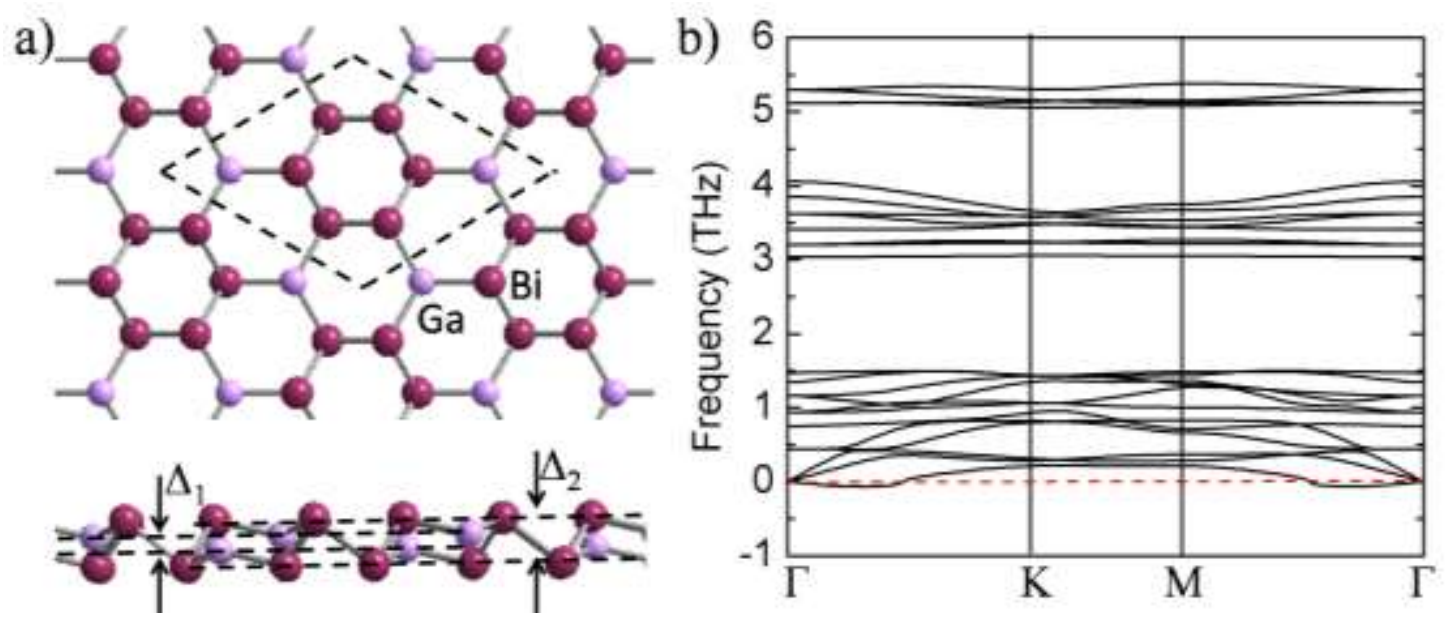
Figure 3. Partial densities of states and band structures obtained without spin-orbit coupling (SO), and with $\mathrm{SO}$ for the XBi sheets: (a) BBi, (b) AlBi, (c) $\mathrm{GaBi}$, and (d) InBi. Fermi level was set to zero energy.
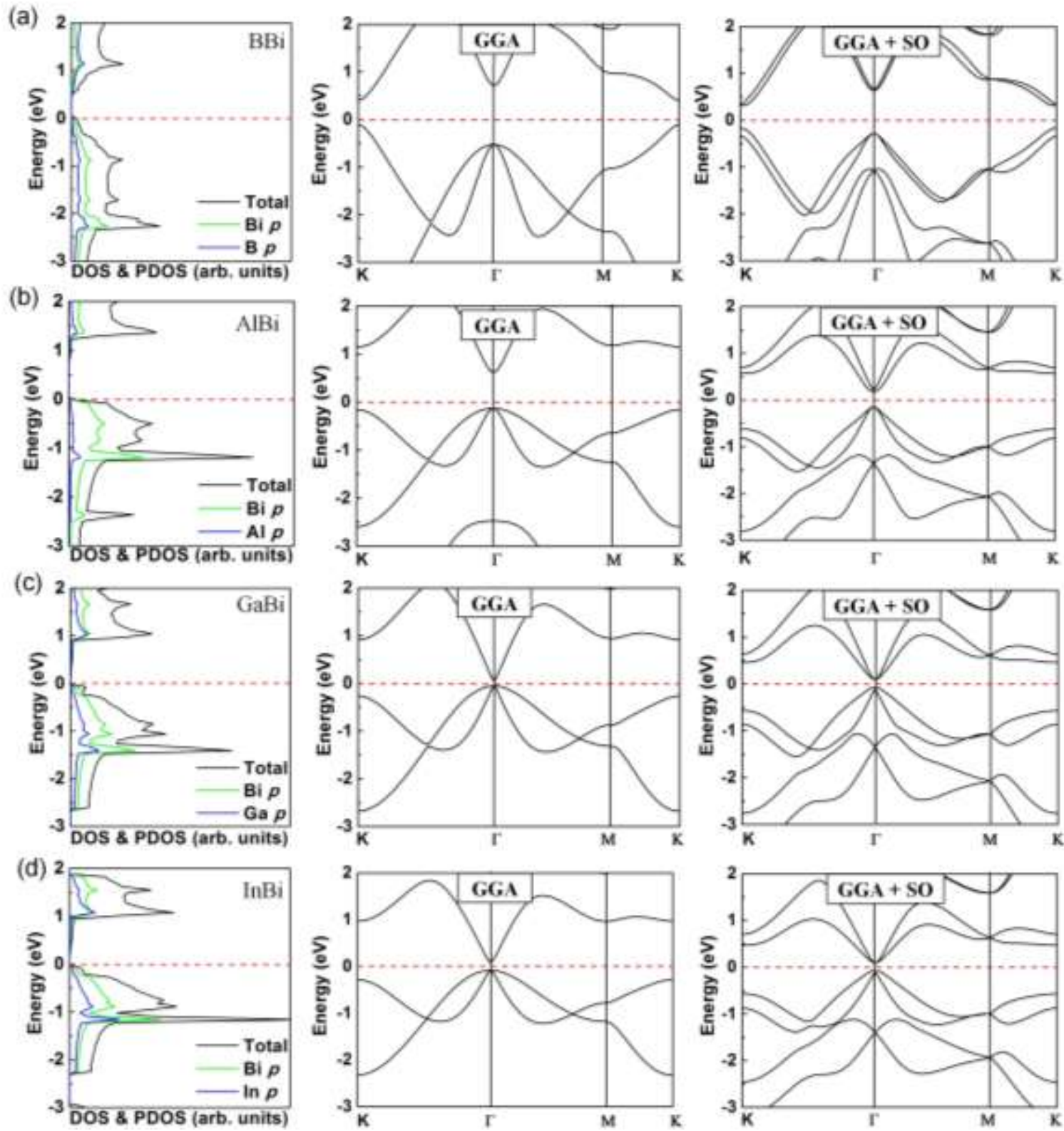
Figure 4. Partial densities of states and band structures obtained without SO and with SO for the $\mathrm{XBi}_{3}$ sheets: (a) $\mathrm{BBi}_{3}$, (b) $\mathrm{AlBi}_{3}$, (c) $\mathrm{GaBi}_{3}$, and (d) $\mathrm{InBi}_{3}$. Fermi level was set to zero energy.
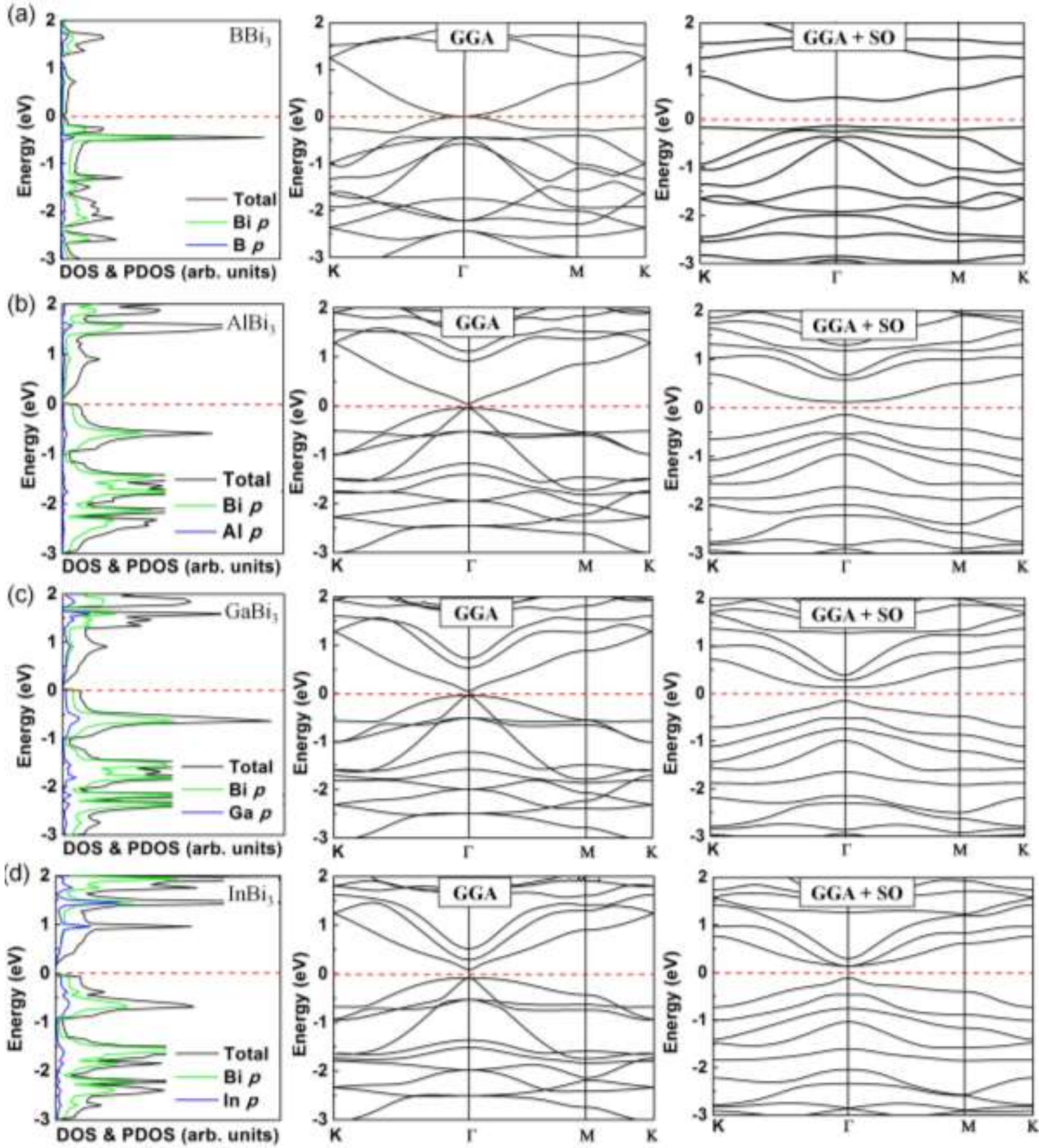
Figure 5. Band structure without and with $\mathrm{SO}$ coupling for (a) $\mathrm{GaBi}$ and (b) $\mathrm{GaBi}_{3}$. The size of the red circles is proportional to the contributions of the s-orbitals to the wave functions.
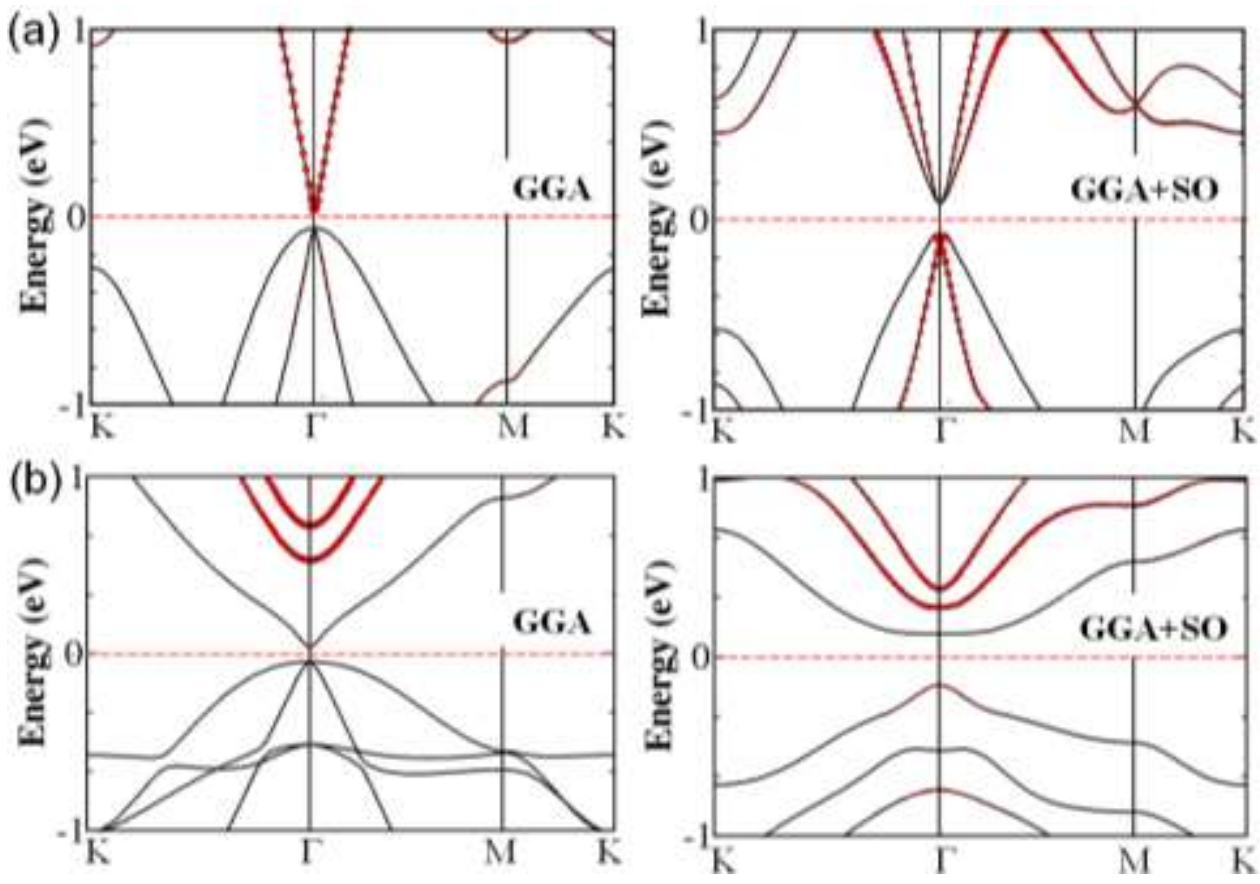
Figure 6. Band inversion strength at the $\Gamma$ point for the XBi sheets.

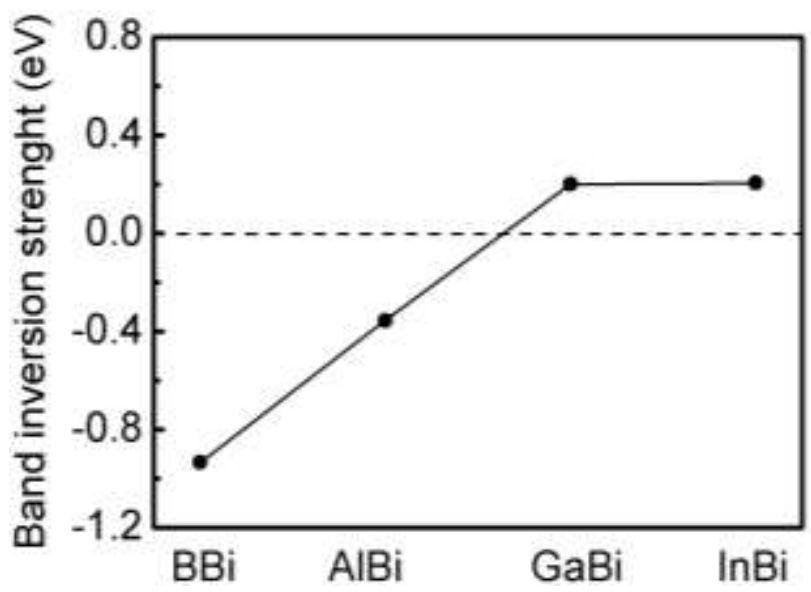

